\title{
Vehicular Messaging In IOT Using Epidemic Routing
}

\author{
Vrushali Pavitrakar \\ Computer Department PVPIT, \\ Pune, India
}

\author{
Navnath Kale \\ Computer Department PVPIT, \\ Pune, India
}

\begin{abstract}
Now a days there are lots of inventions done in vehicles and some may in progress, these inventions help to resolve the challenges caused by the increasing transportation issues. These modern vehicles equipped with a large amount of sensors, actuators and communication devices e.g. GPS. New vehicles have possessed powerful sensing, networking, communication and data processing capabilities and can communicate with other vehicles or exchange information with the external environments over various protocols. This can be done with the help of Vehicular communication systems; these are the networks in which vehicle and roadside units are the communicating node, with each other provide the information, such as safety warnings and traffic information. They can be effective in avoiding accidents and traffic congestion. To solve these issue sending and receiving messages is an important factor. All this things are good when the number of vehicles are less, system must be able to handle traffic spike or sudden demands caused by special events or situations such as sport games or emergencies. This is nothing but a scalability challenge which is going to overcome in this paper using routing protocol called Epidemic routing protocol.
\end{abstract}

Keywords: VCS (Vehicle communication system). VANET (Vehicular Ad Hoc Network), GPS (Global Positioning System), IOT (Internet of Things)

\section{INTRODUCTION}

The inventions in IoT have provided a promising opportunity to address the increasing transportation issues such as heavy traffic, congestion and vehicle safety. (What is IOT? The Internet of Things (IoT) is the network of physical objects, devices, vehicles, buildings and other items which are embedded with electronics, software, sensors, and network connectivity, which enables these objects to collect and exchange data.) Now a day's vehicle builds up with variety of devices like sensors, actuators and communication devices. Vehicular communication systems are networks in which vehicles and roadside units are the communicating nodes, providing each other with information, such as safety warnings and traffic information. They can be effective in avoiding accidents and traffic congestion. The main incentive for VCS is safety and eliminating the excessive cost of traffic collisions. According to World Health Organizations (WHO), road accidents annually cause approximately 1.2 million deaths worldwide. If preventive measures should be taken otherwise road death can be the leading cause of death. In 2001, it was mentioned in a publication that ad hoc networks can be formed by cars and such networks can help overcome blind spots, avoid accidents, etc. This issue can be solve when vehicle talk each other. It is very shocking when we say vehicle can communicate with each other, but yes now a days it is possible. What is vehicle to vehicle communication? - V2V is a technology designed to allow automobiles to "talk" to each other. V2V is currently in active development by General Motors. Now a day's V2V is possible with BMW, Daimler, Honda, Audi, and Volvo. V2V is also known as VANETs. Sometimes around 2020, cars will communicate with each other and alert drivers to roadside hazards ahead. V2V communication technology help to improve safety by allowing vehicles to "talk" to each other and avoid many crashes altogether by exchanging basic safety data, such as speed and position.

With safety data from nearby vehicles, vehicles can identify risks and provide drivers with warnings to avoid other vehicles in common crash types such as rear-end, lane change, and intersection crashes. In this case IoT has received a lot of attention and is expected to bring benefits to numerous application areas including health care, manufacturing and transportation.

\section{LITERATURE SURVEY}

All Wireless technology plays a vital role in vehicular networks. The original idea is the roadside infrastructure and the radio-equipped vehicles could communicate using wireless networks. For more effective routing, Vehicular Ad- hoc Networks (VANET) has been developed. VANETs primarily designed to support the communication between different vehicles $(\mathrm{V} 2 \mathrm{~V})$ and the communication between vehicles and the roadside infrastructures (V2I) [2]. VANET applications focused on improving driver's safety and offered functions such as traffic monitoring and update, emergency warning and road assistance [3].

Now a day's all modern cars equipped with internet help to bring all these ideas. Olariu, Khalil, and Abuelela [3] propose to integrate vehicular networks, sensors, and on-board devices in vehicles to provide more flexibility. The integration of sensors and communication technologies help us to track the changing status of vehicle. IoT explains a future in which a variety of physical objects and devices around us such sensors, radio frequency identification (RFID) tags, GPS devices, and mobile devices will be associated to the Internet and allow these objects and devices to connect, cooperate and communicate within social, environmental, and help to user to reach common goals [4,5]. Speed \& Shingleton [6] propose an idea to use the "unique identifying properties of car registration plates" to connect various things. IoT technologies make it possible to track each vehicle existing location, monitor its movement and predict it future location. An intelligent informatics system (iDrive system) developed by BMW used various sensors and tags to monitor the environment such as tracking the vehicle location and the road condition to provide 
driving directions [7]. Leng and Zhao [8] propose an intelligent internet-of-vehicles system (known as IIOVMS) to collect traffic information from the external environments and to monitor and manage road traffic in real time. Here we proposed a routing protocol which will eventually deliver lots of messages. The epidemic routing protocol able to deliver all messages to its destination without any failure. Epidemic Routing [9] is to distribute messages to hosts, called carriers, within connected portions of ad hoc networks.

\section{SYSTEM ARCHITECTURE}

\subsection{Epidemic Routing System}

Routing protocols allow nodes with wireless adaptors to communicate with other without any pre-existing network infrastructure. Rapidly changing network topology help to deliver messages in the case where there is never a connected path from source destination. Here we are using Epidemic Routing, where random pair-wise exchanges of messages among mobile hosts ensure eventual message delivery. The goals of Epidemic Routing are to: I) maximize message delivery rate, ii) minimize message latency and iii) minimize the total resources consumed in message delivery. Wireless network adaptors in portable computing devices, such as cellular phones, personal digital assistants, and laptops. The goal of this type of protocol is to develop techniques for delivering application data with high probability even when there is never a fully connected path between source and destination or if there is lots of messages. In this way, messages are quickly distributed through connected portions of the network. Epidemic Routing then relies upon carriers coming into contact with another connected portion of the network through node mobility. At this point, the message spreads to an additional island of nodes. Through such transitive transmission of data, messages have a high probability of eventually reaching their destination.

Figure 1 shows Epidemic Routing at a high level, with mobile nodes represented as dark circles and their wireless communication range shown as a dotted circle extending from the source. In Figure 1(a), a source, S, wishes to send a message to a destination, $\mathrm{D}$, but no connected path is available from $\mathrm{S}$ to D. S transmits its messages to its two neighbors, $\mathrm{C} 1$ and $\mathrm{C} 2$, within direct communication range. At some later time, as shown in Figure 1(b), C2 comes into direct communication range with another host, $\mathrm{C} 3$, and transmits the message to it. $\mathrm{C} 3$ is in direct range of D and finally sends the message to its destination.
(1)

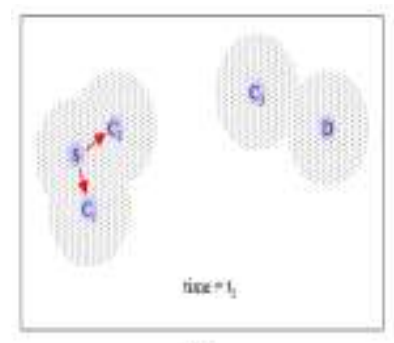

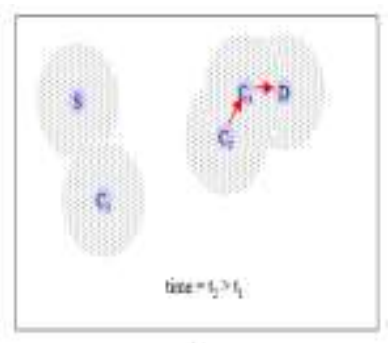

(b)
Fig1. A source, $\mathrm{S}$, wishes to transmit a message to a destination but no connected path is available in part (a). Carriers, C1-C3 are leveraged to transitively deliver the message to its destination at some later point in time as shown in (b).
The goal of Epidemic Routing is: to deliver a message (update) with high probability to a particular host. In fact if there is certain situation where message count is high than regular, in this case also routing protocol should be able to send or receive message eventually. Likewise Epidemic Routing should be able to message broadcast/multicast in partially connected ad hoc networks. The overall goal of Epidemic Routing is to maximize message delivery rate and minimize message delivery latency, while also minimizing the aggregate system resources consumed in message delivery. We explore message delivery rate and resource consumption under a number of different scenarios. Our results show that Epidemic Routing is able to deliver all messages in where existing ad hoc routing protocols fail to delivery some messages because of limited node connectivity. Epidemic Routing delivers $100 \%$ of messages assuming enough per-node buffering to store between $10-25 \%$ of the messages originated in the scenario.

1. Goal:- The goals of Epidemic Routing are to:

- Efficiently distribute messages through partially connected ad hoc networks in a probabilistic fashion,

- Minimize the amount of resources consumed in delivering any single message

- Maximize the percentage of messages that are eventually delivered to their destination

2. Epidemic Routing Protocol: Epidemic Routing supports the eventual delivery of messages based on minimal assumptions like only periodic pair-wise connectivity is required to ensure eventual The Epidemic Routing protocol works as follows.

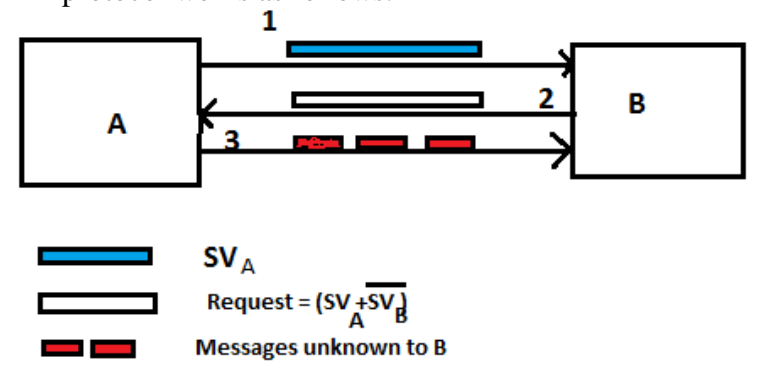

Figure 2: The Epidemic Routing protocol when two hosts, $A$ and $B$, come into transmission range of one another

Each host maintains a buffer consisting of messages that it has originated as well as messages that it is buffering on behalf of other hosts. For efficiency, a hash table indexes this list of messages, keyed by a unique identifier associated with each message. Each host stores a bit vector called the summary vector that indicates which entries in their local hash tables are set. When two hosts come into communication range of one another, the host with the smaller identifier initiates an antientropy session. To avoid redundant connections, each host maintains a cache of hosts that it has spoken with recently. Anti-entropy is not re-initiated with remote hosts that have been contacted within a time period. During anti-entropy, the two hosts exchange their summary vectors to determine which messages stored remotely have not been seen by the local host. In turn, each host then requests copies of messages that it has not yet seen. The receiving host maintains total autonomy in deciding whether it will accept a message. For example, it may determine that it is unwilling to carry messages larger than a given size or destined for certain hosts. We do model a maximum queue size associated with each host, which determines the maximum number of messages a host is willing 
to carry on behalf of other hosts. Figure 2 depicts the message exchange in the Epidemic Routing protocol. Host A comes into contact with Host B and initiates an anti-entropy session. In step one, A transmits it summary vector, and SVA to B. SVA is a compact representation of all the messages being buffered at A. Next, B performs a logical AND operation between the negation of its summary vector, SVB, and SVA. That is, B determines the set difference between the messages buffered at $\mathrm{A}$ and the messages buffered locally at $\mathrm{B}$. It then transmits a vector requesting these messages from $\mathrm{A}$. In step three, A transmits the requested messages to $\mathrm{B}$. This process is repeated transitively when $\mathrm{B}$ comes into contact with a new neighbor.

\subsection{Proposed System Architecture}

Given sufficient buffer space and time, these antientropy sessions guarantee eventual message delivery through such pair-wise message exchange. Epidemic Routing associates a unique message identifier, a hop count, and an optional acknowledgment request with each message. Thus, high priority messages might be marked with a high hop count, while Given that messages are delivered probabilistically in epidemic routing, certain applications may require acknowledgments of message delivery.

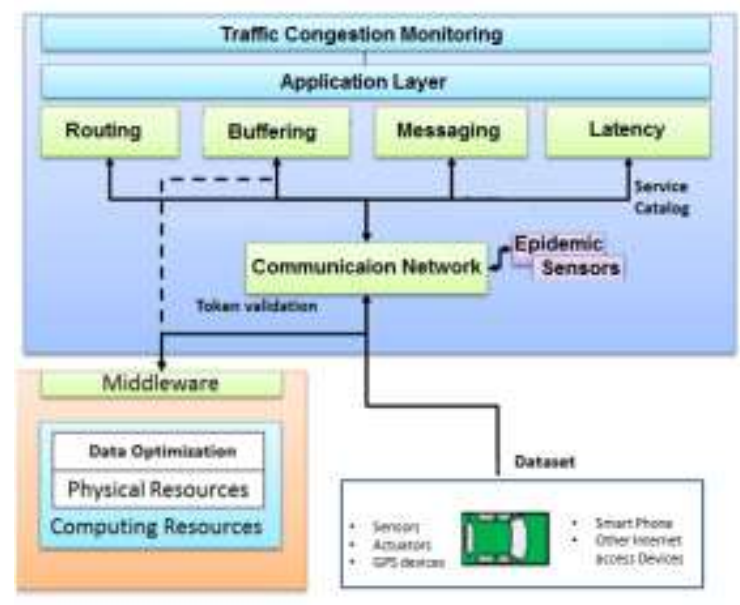

Fig 3: System Architecture

Each host sets a maximum buffer size that it is willing to allocate for epidemic message distribution. The buffer size limits the amount of memory and network resources consumed through Epidemic Routing. In general, hosts will drop older messages in favor of newer ones upon reaching their buffer's capacity. To ensure eventual delivery of all messages, the buffer size on at least a subset of nodes must be roughly equal to the expected number of messages in transit at any given time. Otherwise, it is possible for older messages to be deleted from all buffers before delivery. Fig 3 shows system architecture diagrammatically.

\section{CONCLUSION}

In this paper, we are going to use epidemic routing protocol which will allow confirm message delivery even if there are 100 or 1000 of messages to be deliver in some special cases. Existing ad hoc routing protocols are robust to rapidly changing network topology, but they are unable to deliver packets in the presence of a network partition between source and destination.

Including mobile sensor networks and disaster recovery scenarios, nodes can be spread over wide geographical distances such wide dispersion makes it unlikely that a connected path can always be discovered, because of that it is impossible to perform message delivery using current ad hoc routing protocols. Thus, we introduce Epidemic Routing, where random pair-wise exchanges of messages among mobile hosts ensure eventual message delivery. The goals of Epidemic Routing are to maximize message delivery rate and to minimize message latency while also minimizing the total resources (e.g., memory and network bandwidth) consumed in message delivery. In our case we will show that Epidemic Routing delivers $100 \%$ of eventual message delivery with reasonable resource consumption where existing ad hoc routing protocols are unable to deliver some messages because no end-to-end routes are available.

\section{ACKNOWLEDGMENTS}

I wish to thanks my guide Mr. Navnath kale sir for their guidance and encouragement for this work. I am also thankful to the principle of PVPIT College for providing me the opportunity to embark this project.

\section{REFERENCES}

[1] Wu He, Gongjun Yan and Li Da Xu, Senior Member, IEEE, "Developing Vehicular Data Cloud Services in the IoT Environment.

[2] Wu He, Gongjun Yan and Li Da Xu, Senior Member, IEEE, "Developing Vehicular Data Cloud Services in the IoT Environment.

P. Papadimitratos, A. La Fortelle, K. Evenssen, R. Brignolo, and S. Cosenza, "Vehicular communication systems: Enabling technologies, applications, and future outlook on intelligent transportation," IEEE Communications Magazine, 47(11), 84-95, 2009.

[3] S. Olariu, I. Khalil, and M. Abuelela, "Taking VANET to the clouds," International Journal of Pervasive Computing and Communications, 7(1), pp.721, 2011s

[4] European Commission Information Society. Internet of Things in 2020: a Roadmap for the Future. Available from: www.iot-visitthefuture.eu

[5] L. Atzori, A. Iera, and G. Morabito, "The internet of things: A survey," Computer Networks, 54(15), pp. 2787-2805, 2010.

[6] ]C. Speed, and D. Shingleton, “An internet of cars: connecting the flow of things to people, artefacts, environments and businesses," In Proceedings of the 6th ACM workshop on Next generation mobile computing for dynamic personalised travel planning, pp. 11-12, ACM, 2012.

[7] E. Qin, Y. Long, C. Zhang, and L. Huang, "Cloud Computing and the Internet of Things: Technology Innovation in Automobile Service," In Human Interface and the Management of Information. 
Information and Interaction for Health, Safety, Mobility and Complex Environments (pp. 173-180). Springer Berlin Heidelberg, 2013.

[8] Y. Leng, and L. Zhao, "Novel design of intelligent internet-of-vehicles management system based on cloudcomputing and Internet-of-Things," Proceedings of 2011 International Conference on Electronic and Mechanical Engineering and Information Technology (EMEIT), Vol. 6, pp. 3190-3193, 2011

[9] A. Demers, D. Greene, C. Hauser, W. Irish, J. Larson, S. Shenker, H. Sturgis, D. Swinehart, and D. Terry. Epidemic Algorithms for Replicated Database Maintenance. In Proceedings of the Sixth Symposium on Principles of Distributed Computing, pages 1-12, August 1987 